\title{
Breaking the Transmission Barriers in Ultra-broadband High-capacity Optical Fiber Transmission Systems
}

\author{
L. Galdino, D. Semrau, P. Bayvel \\ Optical Networks Group, Dept. of Electronic \& Electrical Engineering, UCL (University College London), UK \\ *l.galdino@ucl.ac.uk
}

\begin{abstract}
The limitations and practicalities in design of ultra-high-capacity optical transmission systems with transmission bandwidth beyond C+L EDFA are described, together with a deep theoretical understanding of each noise source contribution limiting the overall data throughput.
\end{abstract}

\section{Introduction}

Recent years have seen several landmark transmission results using single mode fibre (SMF) [1] [2] [3] [4] [5] [6] [7] [8]. Advanced methods and concepts employed in the forefront spectrally efficient coherent optical fiber transmission systems, such as probabilistic and geometrical shaping, adaptive digital nonlinear compensation and per channel adaptive code rated combined with advanced high speed electronics, large-effective area / low-loss transmission fibers, and broadband hybrid distributed RamanEDFA amplifier, have resulted in experimental SMF capacity increases as highlighted in Fig.1. The trans-Atlantic and trans-Pacific record data throughput to date is $70.46 \mathrm{Tbit} / \mathrm{s}$ over 7,600 km [1] and $51.5 \mathrm{Tbit} / \mathrm{s}$ over 17,107 km [2]. Theses records have been empowered by the combination of coded modulation with hybrid probabilistic and geometrical constellation shaping with multi-stage nonlinearity compensation including digital back-propagation, fast LMS equalizer and generalized filter, together with a per

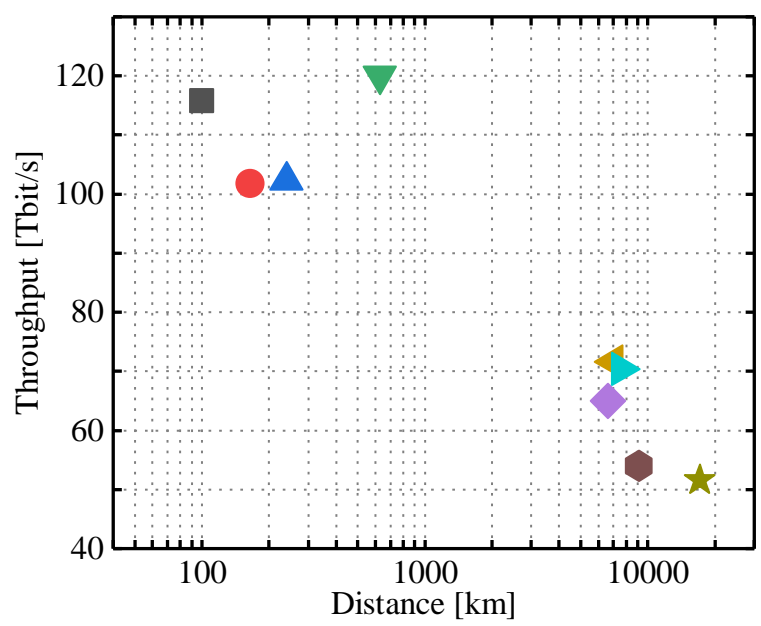

Fig. 1. Records data throughput versus distance. channel adaptive-rate decoding. Despite the advantages of using low noise hybrid distributed Raman-EDFA amplifier (HRE) as demonstrated in [3] most of theses records throughput in trans-Atlantic and trans-Pacific distance was achieved by using $\mathrm{C}+\mathrm{L}$ band EDFA. On the other hand, the records throughout in short, metropolitan transmission distance have been achieved mainly by using amplification techniques that goes beyond C+L band EDFAs. In [4], a continuous-band $100 \mathrm{~nm}$ semiconductor optical amplifier (SOA) enabled a potential SMF capacity of $115.9 \mathrm{Tbit} / \mathrm{s}$ over $100 \mathrm{~km}$. Although the bandwidth is notable, SOAs have a relatively high noise figure compared with distributed Raman amplifiers, so the system performance decreases rapidly with distance. High data throughput in [5] [6] and [7] was achieved by using continuous $90 \mathrm{~nm}$ hybrid distributed Raman-EDFA amplifier, with the world record SMF data throughput of $120 \mathrm{Tbit} / \mathrm{s}$ over $630 \mathrm{~km}$ demonstrated in [7].

Recently, it was demonstrated that transceiver constrained-SNR imposes a significant reduction in throughput for medium transmission links [9]. In this paper we further investigate the impact of transceiver constrained-SNR, limiting the overall transmission system performance. In particularly, the reduction in received SNR due to transceiver noise contribution is studied from short to trans-Atlantic distance.

\section{Transmission System Design}

Fundamental limits of an optical communication system are imposed by a combination of noise from the transceiver subsystem, optical amplifier and optical fibre nonlinearity. An upper bound on the achievable SNR in coherent optical communication systems is set by the noise introduced by the transceiver subsystems [10]. Significant sources of transceiver noise include quantisation noise due to the finite resolution of digital-to-analog converters (DAC) and analogto-digital converters (ADC) as well as noise from the linear electrical amplifiers. During transmission, the achievable SNR is further limited by amplified spontaneous emission noise (ASE) from optical amplifiers in the transmission line and by nonlinear signal distortion, inherent to transmission through silica optical fibres. Therefore, to investigate the individual noise source contribution of each transmitted channel, and the overall system performance, a basic SNR model 
is used. After coherent detection and electronic dispersion compensation, the received signal-to-noise ratio can be calculated as $\mathrm{SNR}_{\mathrm{Total}}=\frac{P}{\kappa P+N P_{\mathrm{ASE}}+N P_{\mathrm{NLI}}}$, with $\kappa=$ $1 / \mathrm{SNR}_{\mathrm{TRX}}$, were $\mathrm{SNR}_{\mathrm{TRX}}$ is the maximum SNR that can be achieved in a given transceiver subsystem, and it can be measured in a back-to-back configuration, $N$ is the number of spans, $P_{\mathrm{ASE}}$ is the accumulated ASE noise power and $P_{\mathrm{NLI}}$ is the nonlinear noise power. The transmission system considered in work is an $312 \times 35$ GBd dual polarization 256ary quadrature amplitude modulation (DP-256QAM). The transmission link is comprise by spans with $70 \mathrm{~km}$ of SMF and hybrid distributed Raman-EDFA amplifier (HRE). Detailed experimental setup description of this system under investigation can be found in [7]. The theoretical calculation of $P_{\mathrm{ASE}}$ and $P_{\mathrm{NLi}}$ was performed using the inter-channel stimulated Raman scattering (ISRS) GN-model [11] and the accuracy of this system modeling compared to the experimental measurements is shown in [12].

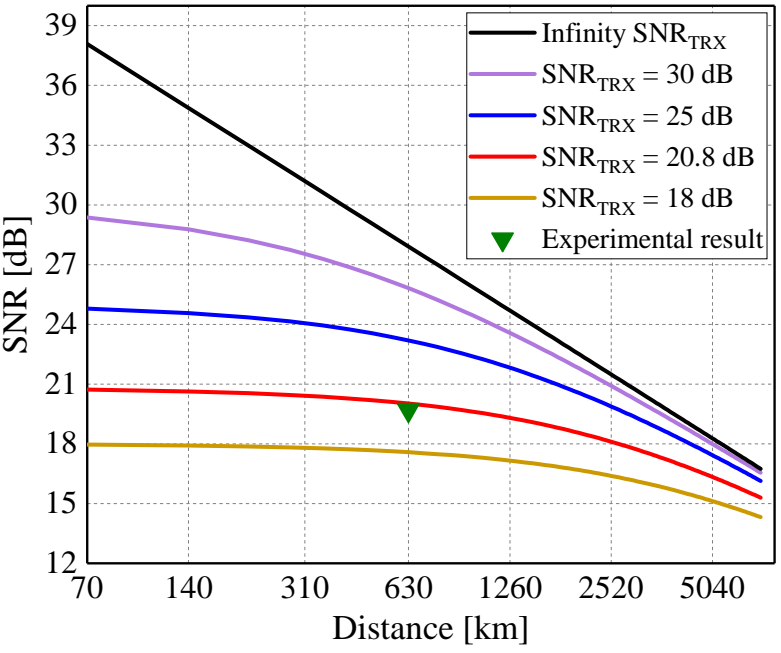

Fig. 2. Received SNR versus distance for different transceiver constrained-SNR.

The plot in Fig. 2 shows the impact of different transceiver-constrained performance $\left(\mathrm{SNR}_{\mathrm{TRX}}\right)$ on the received $\mathrm{SNR}_{\text {Total }}$ over different transmission distances. The green marker is the mean SNR of the 312 channels measured experimentally after $630 \mathrm{~km}$ (full set of results can be found in [7]). This particular experimental system, with a transceiver SNR TRX $_{\text {of }}$ $20.8 \mathrm{~dB}$ has been well modeled with an offset of the model prediction of only $0.4 \mathrm{~dB}$, with a mean $\mathrm{SNR}_{\text {Total }}$ of $19.3 \mathrm{~dB}$ experimentally measured. The black line illustrates the model prediction of the receiver SNR Total when the transceiver subsystem is ideal $\left(\mathrm{SNR}_{\mathrm{TRX}}=\infty\right)$. The model predicts that for an ideal transceiver subsystem, the received $\mathrm{SNR}_{\text {Total }}$ after $630 \mathrm{~km}$ would be $27.9 \mathrm{~dB} ; 8.6 \mathrm{~dB}$ higher compared to the system with a SNR $\mathrm{TRX}_{\text {of }} 20.8 \mathrm{~dB}$. Similar gains in transmission distance can also be observed. For instance, for an ideal transceiver, the model predicts a received $\mathrm{SNR}_{\text {Total }}$ of $19.3 \mathrm{~dB}$ after transmission distance of $3430 \mathrm{~km}$; more than 5-fold increase in distance compared with the system under investigation, that exhibit a $\mathrm{SNR}_{\mathrm{TRX}}$ of $20.8 \mathrm{~dB}$.

As expected, as the transmission distance increases the impact of transceiver limited SNR becomes less significant and the system performance becomes dominated by the nonlinear distortion. High quality signal generation and reception are crucial to improve signal quality and maximize data throughput in medium- and long-haul transmission links.

Support from the Royal Academy of Engineering under the Research Fellowships programme and UK EPSRC TRANSNET EP/R035342/1, is gratefully acknowledged.

\section{References}

[1] T-X. Cai et al., "70.46 Tb/s over 7,600 km in in C+ L Band Using Coded Modulation with Hybrid Constellation Shaping and Nonlinearity Compensation, Proc. OFC, PDP Th5B.2 (2017).

[2] T-X. Cai et al., "51.5 Tb/s Capacity over 17,107 km in C+L Bandwidth Using Single-Mode Fibers and Nonlinearity Compensation, J. Lightw. Technol., 36 (11), (2018).

[3] T-X. Cai et al., " $49.3 \mathrm{~Tb} / \mathrm{s}$ Transmission Over $9100 \mathrm{~km}$ Using C+L EDFA and $54 \mathrm{~Tb} / \mathrm{s}$ Transmission Over $9150 \mathrm{~km}$ Using Hybrid-Raman EDFA, J. Lightw. Technol., 33 (13), (2015).

[4] J. Renaudier et al., "First 100-nm Continuous-Band WDM Transmission System with 115Tb/s Transport over 100km Using Novel UltraWideband Semiconductor Optical Amplifiers," Proc. ECOC, PDP (2017).

[5] A. Sano et al., "102.3-Tb/s C-band extended L-band all Raman transmission over 240 km using PDM-64QAM single carrier FDM with digital pilot tone," Proc. OFC, PDPSC.3 (2012).

[6] D. Qian et al., "101.7-Tb/s (370x294-Gb/s) PDM-128QAM-OFDM Transmission over 3x55-km SSMF using Pilot-based Phase Noise Mitigation," Proc. OFC, PDPB.5 (2011).

[7] M. Ionescu et al., "90 nm C + L Hybrid Distributed Raman/Erbium-Doped amplifier for High Capacity Subsea Transmission," Proc. ECOC,(2018).

[8] A. Ghazisaeidi et al., "Advanced C+L-Band Transoceanic Transmission Systems Based on Probabilistically Shaped PDM-64QAM, J. Lightw. Technol., 35 (7), (2017).

[9] L. Galdino et al., "Impact of Transceiver Subsystems on High-Capacity Optical Transmission, Proc. SPPCom, SpTh2G.1 (2018).

[10] L. Galdino et al., "The Trade-off Between Transceiver Capacity and Symbol Rate," Proc. OFC, W1B.4 (2018).

[11] D. Semrau et al., "The Gaussian Noise Mode in the Presence of Inter-Channel Stimulated Raman Scattering, J. Lightw. Technol., 36 (14), (2018).

[12] L. Galdino et al., " A Per-channel Model for Performance Prediction in Broadband Transmission Systems using Hybrid Distributed Raman-EDFA Amplifier arXiv:1904.01845 (2018). 